\title{
Comparative study of lymphocytes from individuals that were vaccinated and unvaccinated against the pandemic 2009-2011 H1N1 influenza virus in Southern Brazil
}

\author{
Deise Nascimento de Freitas ${ }^{[1]}$, Henrique Ataíde Isaía ${ }^{[1]}$, Andréia Henze ${ }^{[2]}$, Eder Simão ${ }^{[1]}$, \\ Rodrigo Benedetti Gassen ${ }^{[1]}$ and Luiz Carlos Rodrigues Junior ${ }^{[1],[3]}$
}

[1]. Laboratório de Biologia Molecular e Cultivo Celular, Centro Universitário Franciscano, Santa Maria, Rio Grande do Sul, Brazil. [2]. Laboratório de Microbiologia Molecular, Instituto de Ciências da Saúde, Universidade Feevale, Novo Hamburgo, Rio Grande do Sul, Brazil. [3]. Laboratório de Imunologia, Universidade Federal de Ciências da Saúde de Porto Alegre, Porto Alegre, Rio Grande do Sul, Brazil.

\begin{abstract}
Introduction: While no single factor is sufficient to guarantee the success of influenza vaccine programs, knowledge of the levels of immunity in local populations is critical. Here, we analyzed influenza immunity in a population from Southern Brazil, a region with weather conditions that are distinct from those in the rest of country, where influenza infections are endemic, and where greater than $50 \%$ of the population is vaccinated annually. Methods: Peripheral blood mononuclear cells were isolated from 40 individuals. Of these, 20 had received the H1N1 vaccine, while the remaining 20 were unvaccinated against the disease. Cells were stimulated in vitro with the trivalent post-pandemic influenza vaccine or with conserved major histocompatibility complex I (MHC I) peptides derived from hemagglutinin and neuraminidase. Cell viability was then analyzed by [3-(4,5-dimethylthiazol2-yl)-2,5- diphenyltetrazolium bromide)]-based colorimetric assay (MTT), and culture supernatants were assayed for helper T type 1 (Th1) and Th2-specific cytokine levels. Results: Peripheral blood lymphocytes from vaccinated, but not unvaccinated, individuals exhibited significant proliferation in vitro in the presence of a cognate influenza antigen. After culturing with vaccine antigens, cells from vaccinated individuals produced similar levels of interleukin (IL)-10 and interferon (IFN)- $\gamma$, while those from unvaccinated individuals produced higher levels of IFN- $\gamma$ than of IL-10. Conclusions: Our data indicate that peripheral blood lymphocytes from vaccinated individuals are stimulated upon encountering a cognate antigen, but did not support the hypothesis that cross-reactive responses related to previous infections can ameliorate the immune response. Moreover, monitoring IL-10 production in vaccinated individuals could comprise a valuable tool for predicting disease evolution.
\end{abstract}

Keywords: Influenza virus. Vaccine. Peripheral blood lymphocytes. Tropical area.

\section{INTRODUCTION}

While the success of a vaccination program for influenza is determined, at least in part, by the immunogenicity of the circulating strain, it also depends on the level of local immunity, particularly in areas where respiratory infections are historically prevalent. During epidemics of swine origin influenza A (swH1N1), states in Southern Brazil exhibited outbreak characteristics that did not correlate with those of the rest of the country. Throughout the winter, the weather in these states is cold and humid, and there is a high incidence of influenza infections. As such, many people are vaccinated

Corresponding author: Prof. Luiz Carlos Rodrigues Junior. Laboratório de Imunologia/UFCSPA. Av. Sarmento Leite 245, 90050-170 Porto Alegre, Rio Grande do Sul, Brasil.

Phone: 5551 9153-6749; Fax: 5551 3303-9000

e-mail: luizrdrgs@yahoo.com.br

Received 27 May 2015

Accepted 21 July 2015 each year ${ }^{(1)}$. However, despite the administration of a high number of vaccine doses for influenza H1N1, cases of severe swH1N1 infections were more prevalent in these states than in the northern and Northeastern states ${ }^{(2)}$.

Enumeration of the serum titers of antibodies specific to the viral glycoproteins hemagglutinin (HA) and neuraminidase (NA) is a parameter frequently used to evaluate the immune response to influenza ${ }^{(3)}{ }^{(4)}(5)$. Notably, antibodies generated against these glycoproteins in response to a particular strain of influenza do not always guarantee protection to other closely related strains ${ }^{(6)(7)}$. Meanwhile, seroepidemiological studies indicate that there is a lack of widespread pre-existing antibodies against swH1N1 in the general population ${ }^{(8)(9)(10)}$. In addition to antibodies, T cells are also involved in the response to influenza viruses $^{(3)(11)}$. Several studies have demonstrated that infections by, or vaccination to, specific influenza strains can induce the production of cross-reactive $\mathrm{T}$ cells that can potentially recognize conserved epitopes in other strains ${ }^{(12)}$. Recent studies, however, claim that this cross-reactivity does not guarantee an efficient cross-response ${ }^{(11)}$. Furthermore, $\mathrm{T}$ cells that were previously induced by exposure to conserved influenza epitopes exhibit modest memory phenotypes and clonal expansion levels, 
compared to those of naïve cells, upon antigen stimulation ${ }^{(13)}$. Indeed, in a study of Brazilian school children, frequent exposure to seasonal influenza virus strains was not a relevant factor for preventing infection by swH1N1 in $2010^{(14)}$. These findings demonstrate that the relationship between previous exposures to closely related strains of the influenza virus and protection during an epidemic is not applicable to all situations.

To examine the effects of previous exposures to influenza antigens on the immune response to H1N1, we analyzed the response of lymphocytes harvested from the peripheral blood of individuals in Southern Brazil that were either vaccinated or unvaccinated against $\mathrm{H} 1 \mathrm{~N} 1$ to the post-pandemic influenza trivalent vaccine (Fluvac). Additionally, we determined the specificity of the stimulation using influenza H1N1 major histocompatibility class I (MHC class I) conserved epitopes. These analyses were complemented by quantification of the levels of interleukin 10 (IL-10) and interferon (IFN)- $\gamma$ produced by cells after culturing in the presence of influenza antigens. The results of this study enhance our knowledge of the lymphocyte response to influenza in people from an endemic region, and may facilitate the development of improved protocols for influenza immunization and therapies.

\section{METHODS}

\section{Study population}

In total, 40 healthcare professionals between the ages of 20 and 36 years were recruited for this cross-sectional study. Of these, 20 received the influenza vaccine during or after the 2009 H1N1 influenza pandemic, while the remaining 20 had never received the vaccine. Each subject completed a written inquiry regarding their health and vaccination history, and all subjects had a prior history of respiratory infection. Patients suffering from the flu or a cold, and those exhibiting a cough or clinical signs of allergic disorders were excluded from the study.

\section{Antigen stimulation}

Fluvac vaccine formulated using the A/California/7/2009, $\mathrm{A} /$ Perth/16/2009, and B/Brisbane/60/2008 influenza strains was obtained from the Multivacin Immunization Clinic (Santa Maria, RS, Brazil). The dose used for stimulation was determined via a dose-response experiment using concentrations of $0.125,0.25$, 0.03 , and $0.06 \mu \mathrm{g} / \mathrm{well}$. Moreover, cells were stimulated with HA and NA peptides corresponding to the amino acid sequences present in influenza H1N1/California/4/2009, as characterized using immunoinformatics tools ${ }^{(15)}$, which bind multiples alleles of the human leukocyte antigen (HLA) protein of humans (Table 1). Synthetic peptides (Genemed Synthesis, Inc., San Antonio, TX, USA) were diluted in water and then in Roswell Park Memorial Institute (RPMI) 1640 medium (Gibco, Grand Island, NY, USA). The peptide dose used for stimulation was optimized via a dose-response curve experiment using 2.5, $5.0,10,20$, and $25 \mu \mathrm{M}$ concentrations of each of the following epitopes: HA1, LSSVSSFER; HA2, GMVDGWYGY; HA3, MESVKNGTY; NA1, VSFNQNLEY; and NA2, RPWVSFNQN.

\section{Isolation of peripheral blood mononuclear cells}

Eight milliliters of peripheral blood was collected from each volunteer by venipuncture, and samples were stored in ethylenediaminetetraacetic acid (EDTA) tubes (Vacuette ${ }^{\circledR}$, Campinas, SP, Brazil). Peripheral blood mononuclear cells (PBMCs) were then isolated by centrifugation over a $7 \mathrm{~mL}$ Ficoll-Hypaque (Sigma-Aldrich, St. Louis, MO, USA) gradient $(900 \times g, 30 \mathrm{~min})$. After washing with RPMI, cells were viewed microscopically $(40 \times)$, and viability was determined by trypan blue (Sigma, St Louis, MO, USA) exclusion assay analyses. PBMCs were then resuspended in complete culture medium [RPMI-1640, supplemented with $0.55 \mathrm{~g} / \mathrm{L}$ gentamicin, $1 \%$ glutamine, and $10 \%$ fetal bovine serum (FBS)] and adjusted to a concentration of $2 \times 10^{5}$ cells $/ \mathrm{mL}$ with RPMI.

\section{In vitro stimulation of PBMCs}

Peripheral blood mononuclear cells were stimulated in vitro with Fluvac or with HA and NA epitopes. Briefly, $2 \times 10^{5}$ cells were cultured in flat-bottomed 96-well microplates [Techno Plastic Products (TPP) AG, Trasadingen, Switzerland] with RPMI 1640 supplemented with $10 \%$ FBS for $96 \mathrm{~h}$ at $37^{\circ} \mathrm{C}$ in an atmosphere containing $5 \% \mathrm{CO}_{2}$. A $1.5 \%$ concentration of the selective T cell mitogen phytohemagglutinin (PHA; Gibco) was used as a positive control, while medium alone was used as a negative control. To assess in vitro stimulation, the vaccine was diluted in RPMI and added to the cultures at a final concentration of $0.125 \mu \mathrm{g} / \mathrm{well}$. Meanwhile, the HA and NA peptides were each added at final concentrations of $5 \mu \mathrm{M}$, as previously optimized, and the cultures were incubated at $37^{\circ} \mathrm{C}$ with $5 \% \mathrm{CO}_{2}$ for $120 \mathrm{~h}$. For certain experiments, the cultures were also supplemented with 10 international units (IU)/well of human recombinant interleukin (IL)-2 (BD Biosciences ${ }^{\circledR}$, Franklin Lakes, NJ, USA). After antigen stimulation, lymphocyte viability was estimated using an MTT [3-(4,5-dimethylthiazol-2-yl)-2,5- diphenyltetrazolium bromide)]based colorimetric assay ${ }^{(16)}$ (Invitrogen, Carlsbad, CA, USA). The optical density at $560 \mathrm{~nm}\left(\mathrm{OD}_{560}\right)$ of the resulting reactions was measured using a plate reader (Thermoplate ${ }^{\circledR}, \mathrm{RS}$, Brazil).

\section{Analysis of cytokine production}

The concentrations of interferon (IFN)- $\gamma$, IL-10, IL-6, IL-4, and IL-2 within the culture supernatants harvested from stimulated cells were measured using a Cytometric Bead Array (CBA) Th1/ Th2 kit (BD Biosciences ${ }^{\circledR}$ ). Because the cells from each of the 40 individuals were subjected to 10 different stimuli, we chose to pool the supernatants from those exposed to the same stimulus.

\section{Statistical analyses}

Stimulation data were analyzed by repeated measures one-way analysis of variance (ANOVA; non-stimulated vs. stimulated). Multiple comparisons between cytokine levels (PHA mitogen, vaccine, or peptides) were evaluated with Dunnett's and Tukey's post hoc tests to reduce the $\alpha$ error. Differences between variables were also assessed via Student's $t$-tests. Data were expressed as means \pm standard error (SE). Statistical analyses and graphing of the results were performed using GraphPad Prims Pad 4.0 software. The significance level was set at $\mathrm{p} \leq 0.05$. 
TABLE 1 - Conserved hemagglutinin and neuraminidase peptides that bind to multiple human leukocyte antigen class I alleles.

\begin{tabular}{lcccc}
\hline HLA class I Allele & A*0101 & A*0301 & A*2402 & B*0702 \\
\hline $\begin{array}{l}\text { HA } \\
\begin{array}{l}\text { conserved } \\
\text { epitopes }\end{array}\end{array}$ & $\begin{array}{c}\text { LSSVSSFER } \\
\text { GMVDGWYGY } \\
\text { MESVKNGTY }\end{array}$ & $\begin{array}{c}\text { LSSVSSFER } \\
\text { GMVDGWYGY }\end{array}$ & ------- & GMVDGWYGY \\
NA & VSFNQNLEY & VSFNQNLEY & RPWVFNQN & RPWVFNQN \\
$\begin{array}{l}\text { conserved } \\
\text { epitopes }\end{array}$ & & & & \\
\hline
\end{tabular}

HLA: human leukocyte antigen; HA: hemagglutinin; NA: neuraminidase.

\section{Ethical considerations}

The study protocol was approved by the Ethics Committee from Universidade Federal de Santa Maria, Rio Grande do Sul/ Brazil, and experiments were performed in accordance with the ethical standards of the responsible committee on human experimentation (institutional, regional, or national), and in keeping with the Helsinki Declaration of 1964, as revised in 1975, 1983, 1989, 1996, and 2000. All subjects agreed to participate, and each participant provided informed written consent. The protocol approval number is $23081.011864 / 2010-8$.

\section{RESULTS}

Analysis of the response of circulating lymphocytes from vaccinated and unvaccinated individuals to Fluvac antigens

Peripheral blood mononuclear cells from fifteen vaccinated individuals were isolated and stimulated, as described in the Material and Methods. Because a dose-dependent increase in cell viability was not observed within a range of $0.06 \mu \mathrm{g}$ to $0.125 \mu \mathrm{g}$ (Figure 1A), a dosage of $0.125 \mu \mathrm{g}$ was used for all subsequent experiments. Meanwhile, the absorbance value obtained for the PHA-treated cells in the MTT assay was threefold higher than that for the unstimulated cells, indicating that this compound comprised a valid positive control (Figure 1B).

Next, we examined the in vitro response of PBMCs from vaccinated and unvaccinated populations to stimulation with the Fluvac vaccine. As expected, cells from the vaccinated group exhibited significantly higher viability than unstimulated cells (negative control) upon treatment with Fluvac $(0.562 \pm 0.032$ vs. $0.363 \pm 0.017$, respectively; $p=0.0019$; Figure 1C). While the viability of the cells from the unvaccinated group $(0.471 \pm$ $0.029)$ was also higher than that of the negative control cells $(0.363 \pm 0.017)$, this difference was not statistically significant.

Antigen-experienced lymphocytes produced during previous infections typically increase the expression of IL-2 receptor upon re-stimulation. To examine whether cells produced in response to vaccination contribute to in vitro stimulation, we evaluated the response of cells from the vaccinated group to stimulation with Fluvac and IL-2. No significant change in viability was observed in cells from the vaccinated group after treatment with IL-2, compared with that observed in cells stimulated with Fluvac alone (Figure 1D). Similarly, while PBMCs from the unvaccinated group treated with IL-2 exhibited higher levels of stimulation than the negative control cells, this difference was not significant (data not shown). Lastly, there was no difference in the level of lymphocyte stimulation obtained by exposure to Fluvac between the males and females of the vaccinated population (Figure 1E).

Together, these results indicate that, in the population studied, only those individuals that were vaccinated with the monovalent influenza post-pandemic vaccine contained blood circulating lymphocytes that could be promptly stimulated with antigens from the post-pandemic flu vaccine.

\section{Quantification of IFN- $\gamma$ and IL-10 production by lymphocytes from the vaccinated and unvaccinated populations}

Evaluation of lymphocyte viability alone is not a suitable method for predicting the lymphocyte response. Therefore, to complement the MTT assay results, we evaluated the cytokine profile induced by in vitro stimulation of PBMCs from vaccinated and unvaccinated individuals. Specifically, we measured the levels of helper T type 1 (Th1) [interferon gamma (IFN)- $\gamma$; interleukin-2 (IL-2); interleukin-6 (IL-6) and tumor necrosis factor (TNF)- $\alpha]$ and helper $\mathrm{T}$ type 2 (Th2) (IL-4, IL-5 and IL-10) specific cytokines in the supernatants from stimulated cells. There were no differences in the levels of IL-2, IL-6, TNF- $\alpha$, IL-4, or IL-5 between the stimulated and negative control cells from either the vaccinated or the unvaccinated group (data not shown). Conversely, there was a dose-dependent increase in the level of IFN- $\gamma$ secretion from the Fluvac-stimulated cells of the vaccinated group, compared to that observed in cells treated with media alone (negative control; Figure 2A). Similar results were obtained using the cells of the unvaccinated group. Surprisingly, however, there were higher levels of IFN- $\gamma$ in the supernatants harvested from the unvaccinated group than in those harvested from the vaccinated group after stimulation with 0.03 and $0.06 \mu \mathrm{g}$ Fluvac.

As observed for IFN- $\gamma$, cells from both the vaccinated and unvaccinated groups produced higher amounts of IL-10 in vitro than did the negative control cells after stimulation with Fluvac (Figure 2B). Notably, while similar levels of IFN- $\gamma$ and IL-10 were produced by the cells from the vaccinated group at each dose tested (Figure 2C), the cells in the unvaccinated group produced higher levels of IFN- $\gamma$ than IL-10 at all doses tested 


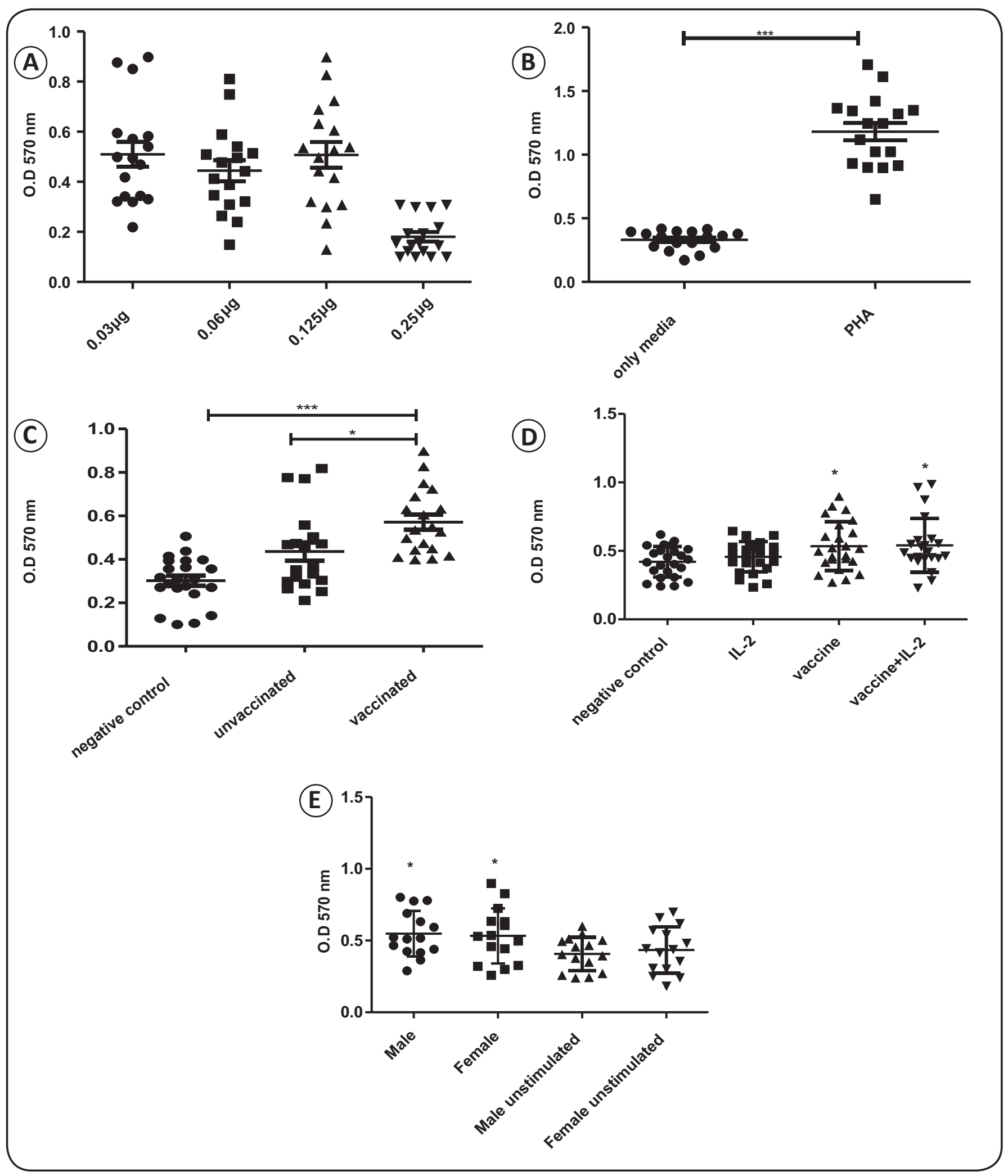

FIGURE 1 - Stimulation of PBMCs from unvaccinated or vaccinated individuals with Fluvac antigens in vitro. A: Dose response analysis $(0.03 \mathrm{~g}$ to $0.25 \mu \mathrm{g})$. B: Comparison of the viability of cells treated with the positive control (PHA) with that of unstimulated cells (media only). C: Viability of PBMCs stimulated with Fluvac (0.125 $\mu$ g). D: Viability of PBMCs from the vaccinated group after stimulation with IL-2 and/or Fluvac. E: Viability of unstimulated PBMCs and of Fluvac-stimulated PBMCs harvested from vaccinated males or females. Results were analyzed by one-way ANOVA with Dunnett's and Tukey's post hoc multiple comparison tests. OD: optic density; PBMCs: peripheral blood mononuclear cells; PHA: phytohemagglutinin; IL-2: interleukin-2; ANOVA: analysis of variance. $* \mathrm{p}<0.01 . * \mathrm{p}<0.05 . * * \mathrm{p}<0.001$. 


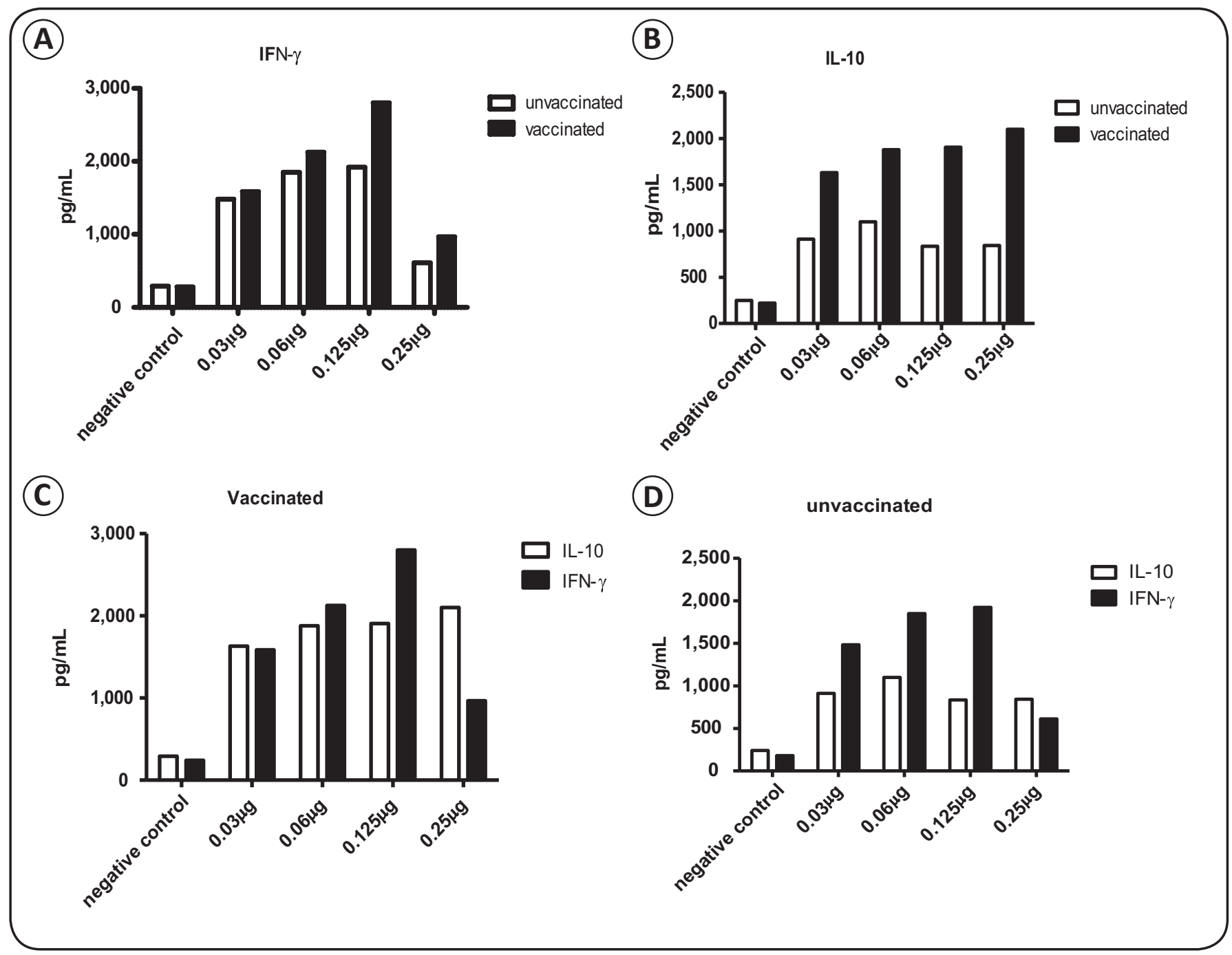

FIGURE 2 - Quantification of the levels of IFN- $\gamma$ and IL-10 in supernatants from PBMCs stimulated with Fluvac or with HA or NA peptides. A: Quantification of IFN- $\gamma$ in the supernatants harvested from the vaccinated and unvaccinated populations after stimulation with whole vaccine (samples were pooled). B: Quantification of IL-10 in the supernatants harvested from the vaccinated and unvaccinated groups after stimulation with Fluvac (samples were pooled). C: and D: Quantification of IFN- $\gamma$ and IL-10 in the supernatants harvested from the (C) unvaccinated and (D) vaccinated groups after stimulation with Fluvac (samples were pooled). OD: optic density, IFN- $\boldsymbol{\gamma}$ : interferon gamma; IL-10: interleukin; PBMCs: peripheral blood mononuclear cells; HA: hemagglutinin; NA: neuraminidase.

(Figure 2D). These data indicate that stimulation of PBMCs from vaccinated individuals with Fluvac antigens results in a balance in the production of IL-10 and IFN- $\gamma$, which is not achieved by unprimed (unvaccinated) cells.

\section{Determination of the specificity of the immune response of lymphocytes from the vaccinated group}

The trivalent vaccine used for the stimulation studies was composed of antigens from two strains of influenza A (A/California/7/2009 and A/Perth/16/2009) and one strain of influenza B (B/Brisbane/60/2008); however, the individuals in the vaccination group were vaccinated with the monovalent vaccine, which contains only antigens from swH1N1 (A/California). To determine whether the A/California/4/2009 peptides, which were previously characterized as ligands for multiple HLA alleles, were present in the Fluvac vaccine, we analyzed the crystal structures of both HA and NA. The HA1, HA2, HA3, NA1, and NA2 peptides from A/California/4/2009 were present in A/California/7/2009 (trivalent vaccine). Furthermore, while the HA2 peptide was conserved in A/ Perth/16/2009 and $\mathrm{A} / \mathrm{California} / 7 / 2009$, this region was structurally different from that in A/California/4/2009. Meanwhile, there was no peptide similarity among other influenza strains (Figure 3).

To evaluate whether cells from the vaccinated and unvaccinated groups were capable of responding to peptides from swH1N1, cells were stimulated with the HA1, HA2, HA3, NA1, and NA2 peptides in vitro. The concentration 
Hemagglutinin

\section{A/California/7/2009}

(A)

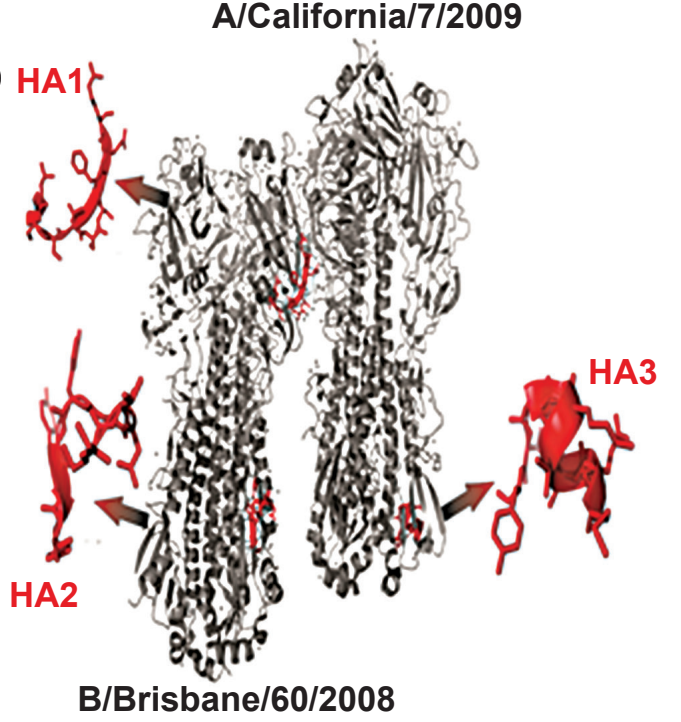

(B)

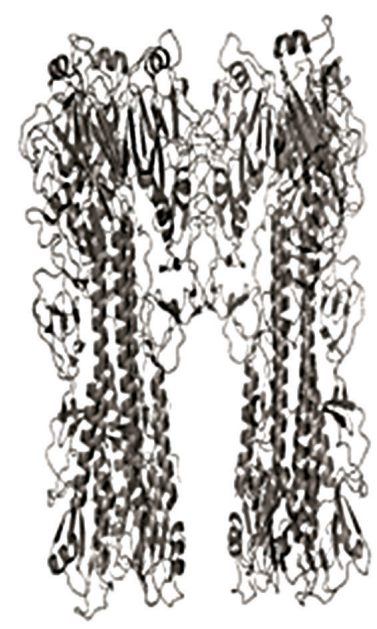

(C)

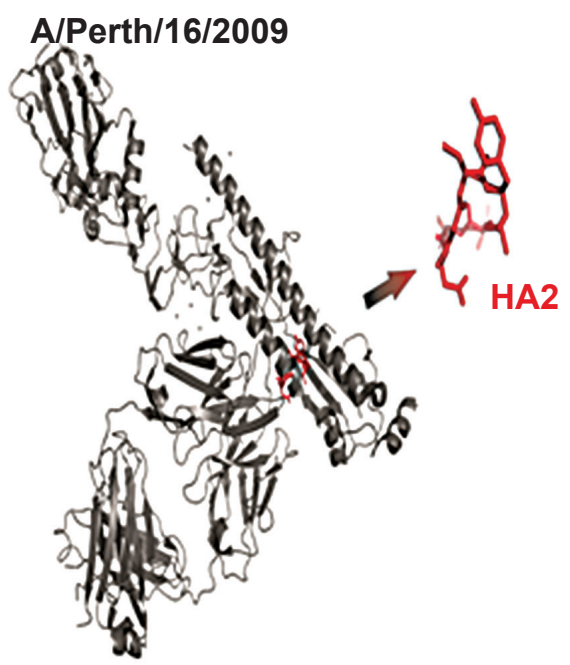

Neuraminidase

A/California/7/2009

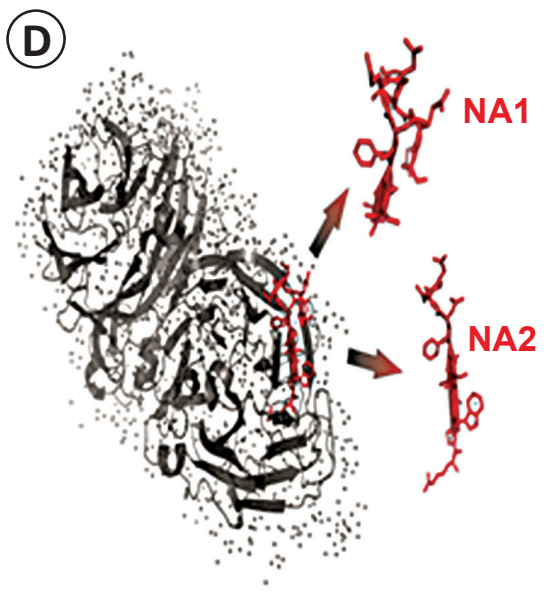

B/Brisbane/60/2008

(E)

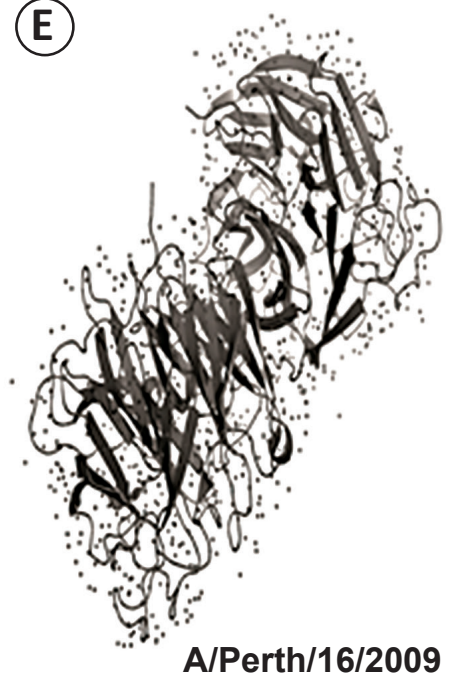

(F)

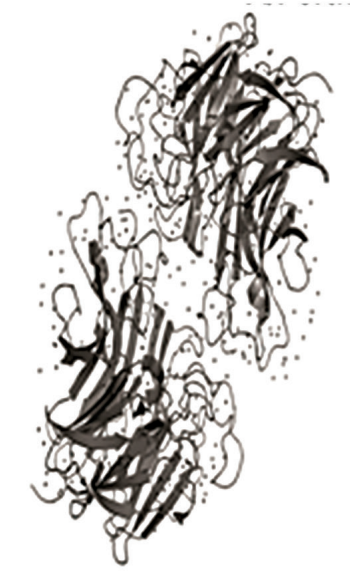

FIGURE 3 - Crystal structure images of HA and NA indicating the location of the peptides used in this study. A: HA1, HA2, and HA3 peptides from influenza $A / C a l i f o r n i a / 7 / 2008$, and $B$ : B/Brisbane/60/2008. C: HA2 peptide from influenza A/Perth/16/2009. D: NA1 and NA2 peptides from influenza A/California/7/2008, E: B/Brisbane/60/2008, and F: A/Perth/16/2009. The structures were built using sequences extracted from the PDB database and the PyMOL molecular graphics system. HA: hemagglutinin; NA: neuraminidase; PDB: protein data bank. 
of HA and NA peptides used for stimulation was optimized by first examining the effects of a range of concentrations $(2.5 \mu \mathrm{M}$ to $40 \mu \mathrm{M})$ on cell viability. As no significant differences were observed among the doses tested (data not shown), a dose of $2.5 \mu \mathrm{M}$ was used for each peptide in all subsequent experiments. Cells from the vaccinated group exhibited higher levels of viability after treatment with each of the HA or NA peptides than with media alone (Figure 4A). In contrast, neither the HA nor the NA peptides enhanced the viability of cells in the unvaccinated group compared to unstimulated cells (Figure 4B).

The influence of IL-2 on the stimulation of lymphocytes was also tested for each epitope. While cells from the vaccinated group were significantly stimulated by both the HA and NA peptides in the presence of IL-2 (Figure 4C), there was no improvement in the stimulation index of cells from the unvaccinated group after stimulation with HA and NA in the presence of IL-2 (Figure 4D). These results indicate that the stimulatory response observed in PBMCs from the vaccinated group was due to the exposure of these individuals to swH1N1 cognate antigens via vaccination, and not to exposure to antigens from other influenza viruses.

\section{DISCUSSION}

Despite numerous efforts to develop an effective vaccine for combating influenza infections, the disease remains a serious health problem, causing frequent epidemics. During the 2009-2011 swH1N1 pandemic, the near total absence of neutralizing antibodies within the general population should have led to a catastrophic influenza pandemic; however, the outcome was less devastating than originally predicted ${ }^{(17)}$. One possible explanation for this is that $T$ cells generated in response to previous influenza vaccinations or infections may have provided heterosubtypic pre-immunity. Here, we demonstrated that peripheral blood lymphocytes harvested from individuals in Southern Brazil that were previously vaccinated with the swH1N1 monovalent vaccine exhibited significant stimulation in vitro in response to antigens from the new trivalent vaccine Fluvac. Specifically, our results indicate that the lymphocytes responded to an antigen from influenza strain $\mathrm{A} /$ California/7/2009 that was present in the trivalent vaccine. Previous studies demonstrated that influenza A virus-specific

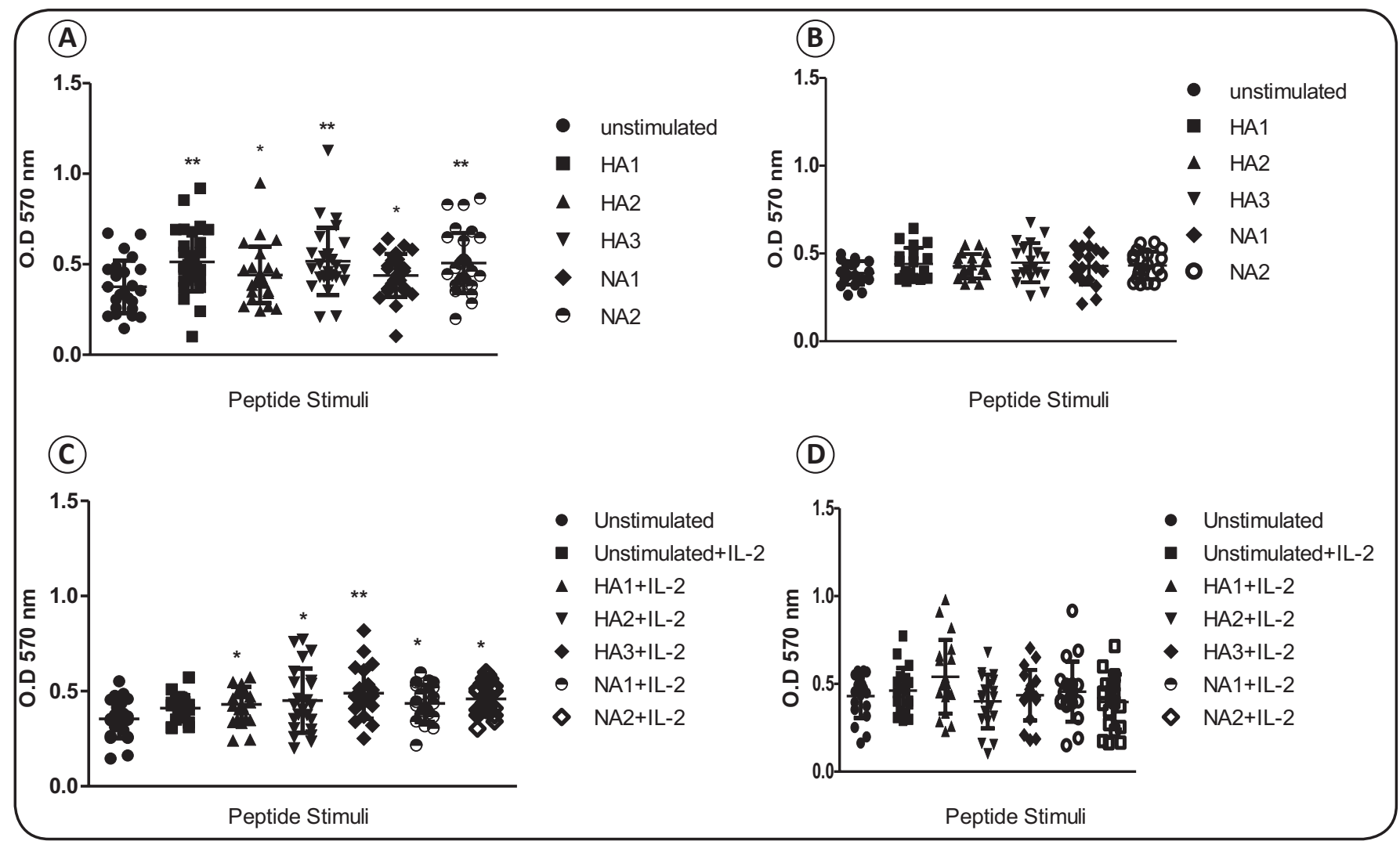

FIGURE 4 - Stimulation of PBMCs from unvaccinated or vaccinated individuals with HA and NA epitopes. A and B: PBMCs from the vaccinated (A) and unvaccinated (B) groups were stimulated with a 5 $\mu$ M concentration of the HA1, HA2, HA3, NA1, or NA2 influenza A conserved peptides. C and D: PBMCs from the (C) unvaccinated and (D) vaccinated groups were stimulated with a $5 \mu \mathrm{M}$ concentration of HA1, HA2, HA3, NA1, or NA2 influenza A conserved peptides, as well as IL-2. Results were analyzed by one-way ANOVA with Dunnett's post hoc multiple comparison tests. OD: optic density; PBMCs: peripheral blood mononuclear cells; HA: hemagglutinin; NA: neuraminidase; ANOVA: analysis of variance. ${ }^{*} \mathrm{p}<0.01,{ }^{* *} \mathrm{p}<0.05$. ${ }^{* * *} \mathrm{p}<0.001$. 
$\mathrm{CD}^{+} \mathrm{T}$ lymphocytes persist for prolonged periods within the peripheral blood of adult subjects ${ }^{(18)(19)(20)}$. These observations corroborate our results, as we examined lymphocytes from subjects that were vaccinated against influenza after the swH1N1 epidemic and the production of an swH1N1-specific vaccine. Furthermore, as in these recent studies, we detected an IFN- $\gamma$ mediated recall response in lymphocytes after stimulation with a cognate antigen. Other studies of distinct populations have also demonstrated that vaccination to influenza provides longterm immunity; however, these studies typically evaluated cell frequencies or the levels of cytokine production by certain cell types such as IFN- $\gamma^{+} \mathrm{IL}-2^{+}$cells, TNF- $\alpha \mathrm{CD} 4^{+} \mathrm{T}$ cells, $\mathrm{CD} 107^{+}$ $\mathrm{CD} 8^{+} \mathrm{T}$ cells, and IFN $\gamma^{+} \mathrm{CD} 49^{+} \mathrm{CD} 8^{+} \mathrm{T}$ cells stimulated in the presence of influenza peptide antigens ${ }^{(21)}$.

Numerous studies have demonstrated that previous influenza vaccinations, as well as infection by seasonal influenza or other influenza virus strains, can generate antibodies and lymphocytes that are cross-reactive to another strain ${ }^{(22)(23)(24)}$. However, the results of our MTT-based cell viability analyses do not support the hypothesis that previous exposures to influenza antigens result in improved responses to other closely related influenza antigens. The influence of previous exposures on the response to influenza is likely more evident, however, when examining cell markers and cytokine levels. In a previous study, Iorio et al. detected blood circulating cells that were reactive against influenza A(H1N1) 2009 antigens in unvaccinated individuals. This group also demonstrated that in vitro re-stimulation of lymphocytes from subjects immunized with a vaccine composed of antigens from strains A/Wisconsin/67/05, A/ Solomon Island/3/06, and B/Malaysia/2506/04 with noncognate antigens resulted in nearly a three-fold increase in the numbers of $\mathrm{CD} 9^{+} \mathrm{CD}^{+}$and $\mathrm{CD} 69^{+} \mathrm{CD} 8^{+} \mathrm{T}$ cells present within the blood. Moreover, stimulation of peripheral $\mathrm{CD} 4^{+}$and $\mathrm{CD} 8^{+} \mathrm{T}$ cells from people who received the monovalent vaccine with pandemic $\mathrm{A} / \mathrm{H} 1 \mathrm{~N} 1$ peptides resulted in a significant proliferative response in $\mathrm{CD} 8^{+}$cells.

Because the immune response is influenced by sexual hormones ${ }^{(25)}$, we also compared the response of lymphocytes from males and females after stimulation with influenza antigens; however, nearly identical stimulation patterns were observed in the cells from both populations, indicating that gender does not influence the immune response to influenza.

SwH1N1 infection and vaccination induce different patterns of cytokine production, depending on the disease severity or the type of antigen used ${ }^{(2)}$. We observed an increase in IL-10 production in the cells from the vaccinated group, compared to those from unvaccinated individuals, indicating that circulating cells that are reactive against influenza antigens are programed to produce IL-10 upon the second exposure to a specific antigen. By comparing IL-10 and IFN- $\gamma$ production within each group, we observed a balance between the two cytokines in the vaccinated group, while the cells in the unvaccinated group produced higher levels of IFN- $\gamma$ than IL-10. Meanwhile, cells from the unvaccinated group produced higher levels of IFN- $\gamma$ in response to Fluvac than did the cells from the vaccinated group. $\mathrm{CD}^{+} \mathrm{T}$ cells are an important source of IL-10, which is essential for regulatory $\mathrm{T}$ cell (Treg) biology, particularly natural $\mathrm{CD} 4^{+} \mathrm{CD} 25^{+} \mathrm{Foxp}^{+} \mathrm{Treg}^{(27)}$. In a previous study, there was an increase in the frequency of $\mathrm{CD}^{+} \mathrm{CD} 25^{+}$and $\mathrm{CD} 4^{+}$ Foxp $3^{+}$cells in the peripheral blood, followed by an increase in the plasma levels of IL-10, of individuals vaccinated against different strains of influenza ${ }^{(28)}$. In addition, Yu et al detected elevated levels of IL-6 and IL-10 in the sera of patients with severe H1N1 infection during the 2009 epidemic. This increase in IL-10 production was also observed in the blood of subjects that were recently immunized with the $\mathrm{H} 1 \mathrm{~N} 1$ vaccine $^{(29)}$, as well as in the pulmonary tissue of mice experimentally infected with influenza A/California/04/09(30)(31).

After examining the effects of Fluvac antigens, we used influenza $\mathrm{CD} 8^{+}$-conserved peptides to investigate whether at least part of the observed response was generated against influenza A-specific antigens. Significant stimulation was observed in cells from the vaccinated group to the HA1, HA2, HA3, NA1, and NA2 peptides; however, there were no significant differences in the levels of viability between the cells stimulated with distinct peptides. A recent study demonstrated that the variability observed in the response to influenza is not due to differences between antigens, but due to differences between individuals or groups of individuals. This finding indicates that previous exposures to particular antigens do not necessarily determine the response to another antigen ${ }^{(32)}$. As such, two conclusions can be drawn from our results obtained using influenza peptides: first, the clonal response of lymphocytes generated in response to the monovalent vaccine can be rescued from the peripheral blood, even after a long period, using the trivalent vaccine; second, this clonal response is not specific to a particular epitope, as exposure to all HA and NA peptides tested induced a response. Other studies have recently shown that $\mathrm{CD} 4^{+}$and $\mathrm{CD} 8^{+} \mathrm{T}$ cells induced by exposure to seasonal influenza virus can cross-recognize internal peptides from H1N1. Such cross-reactive T cells might mediate a heterosubtypic immunity that confers protection against symptomatic illness in individuals lacking pre-existing humoral immunity to the pandemic virus ${ }^{(33)(34)}$. Indeed, Sridhar and collaborators (2012) detected high frequencies of crossreactive $\mathrm{CD} 8^{+} \mathrm{IFN}-\gamma^{+} \mathrm{IL}-2^{+} \mathrm{T}$ cells against live swH1N1 virus in individuals that were seronegative for $\operatorname{swH} 1 \mathrm{~N} 1^{(35)}$.

Our findings indicate that H1N1-reactive blood circulating lymphocytes are prevalent within vaccinated but not unvaccinated individuals, independent of previous infection, from southern Brazil, where there is a high incidence of influenza infections, resulting in background immunity. Furthermore, they demonstrate that effector lymphocytes from vaccinated and unvaccinated individuals exhibit distinct cytokine production profiles. Specifically, upon re-exposure to influenza antigens, the lymphocytes from vaccinated individuals likely mediate a mild inflammatory response that is regulated by IL-10. Conversely, the lymphocytes from unvaccinated individuals likely modulate a robust inflammatory response via the elevated production of IFN- $\gamma$. Meanwhile, there are several limitations to this study. Most notably, the number of subjects tested in this study was relatively low. Following the H1N1 pandemic, many individuals were vaccinated, and it was 
therefore difficult to find unvaccinated subjects. In addition, we were unable to examine a group of infected subjects, as there were no cases of $\mathrm{H} 1 \mathrm{~N} 1$ reported in the area studied, likely due to the high proportion of vaccinated individuals. However, the findings of this study can be used to predict and design more efficient strategies for influenza immunization and therapy by identifying by considering at-risk groups and endemic areas.

\section{ACKNOWLEDGMENTS}

Fundação de Amparo a Pesquisa no Rio Grande do Sul (FAPERGS) for financial support trough the Program for Research in Respiratory Infection (PPIR). Conselho Nacional de Desenvolvimento Científico e Tecnológico (CNPq).

\section{CONFLICT OF INTEREST}

The authors declare that there is no conflict of interest.

\section{FINANCIAL SUPPORT}

\section{Fundação de Amparo a Pesquisa no Rio Grande do Sul.}

\section{REFERENCES}

1. Cerbino Neto J, Penna GO, Werneck GL. Regional differences in mortality associated with pandemic influenza A H1N1 in Brazil. Cad Saude Publica 2013; 29:189-194.

2. Domingues CM, de Oliveira WK. Uptake of pandemic influenza (H1N1)-2009 vaccines in Brazil, 2010. Vaccine 2012; 30:47444751.

3. Hancock K, Veguilla V, Lu X, Zhong W, Butler EN, Sun H, et al. Cross-reactive antibody responses to the 2009 pandemic H1N1 influenza virus. N Engl J Med 2009; 361:1945-1952.

4. Miller E, Hoschler K, Hardelid P, Stanford E, Andrews N, Zambon M. Incidence of 2009 pandemic influenza A H1N1 infection in England: a cross-sectional serological study. Lancet 2010; 375:1100-1108.

5. Gilbert GL, Cretikos MA, Hueston L, Doukas G, O'Toole B, Dwyer DE. Influenza A (H1N1) 2009 antibodies in residents of New South Wales, Australia, after the first pandemic wave in the 2009 southern hemisphere winter. PLoS One 2010; 5:e12562.

6. Tandale BV, Pawar SD, Gurav YK, Parkhi SS, Mishra AC. Antibody persistence after pandemic H1N1 2009 influenza vaccination among healthcare workers in Pune, India. Hum Vaccin Immunother 2013; 9:125-127.

7. Pauksens K. Long-term follow-up in patients with HIV vaccinated with pandemic influenza A (H1N1)/09 AS03-adjuvanted split virion vaccine and seasonal trivalent influenza split virion vaccine. Infect Ecol Epidemiol 2013; 3:10.3402/iee.v3i0.20766.

8. Vergara-Alert J, Argilaguet JM, Busquets N, Ballester M, MartinValls GE, Rivas R, et al. Conserved synthetic peptides from the hemagglutinin of influenza viruses induce broad humoral and T-cell responses in a pig model. PLoS One 2012; 7:e40524.

9. Buricchi F, Bardelli M, Malzone C, Capecchi B, Nicolay U, Fragapane E, et al. Impact of preexisting memory to seasonal A/H1N1 influenza virus on the immune response following vaccination against avian A/H5N1 virus. Eur J Immunol 2013; 43:641-648.

10. Hoschler K, Thompson C, Andrews N, Galiano M, Pebody R, Ellis J, et al. Seroprevalence of influenza A(H1N1)pdm09 virus antibody, England, 2010 and 2011. Emerg Infect Dis 2012; 18:18941897.

11. Tu W, Mao H, Zheng J, Liu Y, Chiu SS, Qin G, et al. Cytotoxic $\mathrm{T}$ lymphocytes established by seasonal human influenza crossreact against 2009 pandemic H1N1 influenza virus. J Virol 2010; 84:6527-6535.

12. Altenburg AF, Rimmelzwaan G, de Vries RD. Virus-specific T cells as correlate of (cross-) protective immunity against Influenza. Vaccine 2015; 33:500-506.

13. Yang J, James E, Gates TJ, DeLong JH, LaFond RE, Malhotra U, et al. CD4+ T cells recognize unique and conserved 2009 H1N1 influenza hemagglutinin epitopes after natural infection and vaccination. Int Immunol 2013; 25:447-457.

14. Guatura SB, Watanabe AS, Camargo CN, Passos AM, Parmezan SN, Tomazella TK, et al. Surveillance of influenza A H1N1 2009 among school children during 2009 and 2010 in São Paulo, Brazil. Rev Soc Bras Med Trop 2012; 45:563-566.

15. De Groot AS, Ardito M, McClaine EM, Moise L, Martin WD. Immunoinformatic comparison of T-cell epitopes contained in novel swine-origin influenza A (H1N1) virus with epitopes in 20082009 conventional influenza vaccine. Vaccine 2009; 27:5740-5747.

16. Mosmann T. Rapid colorimetric assay for cellular growth and survival: application to proliferation and cytotoxicity assays. J Immunol Methods 1983; 65:55-63.

17. Girard MP, Tam JS, Assossou OM, Kieny MP. The 2009 A (H1N1) influenza virus pandemic: A review. Vaccine 2010; 28:4895-4902.

18. McMaster SR, Gabbard JD, Koutsonanos DG, Compans RW, Tripp RA, Tompkins SM, et al. Memory T cells generated by prior exposure to influenza cross react with the novel H7N9 influenza virus and confer protective heterosubtypic immunity. PLoS One 2015; 10:1-11.

19. Li J, Arévalo MT, Chen Y, Chen S, Zeng M. T-cell-mediated crossstrain protective immunity elicited by prime-boost vaccination with a live attenuated influenza vaccine. Int J Infe Dis 2014; 27:37-43.

20. Landry N, Pillet S, Favre D, Poulin JF, Trépanier S, Yassine-Diab $\mathrm{B}$, et al. Influenza virus-like particle vaccines made in Nicotiana benthamiana elicit durable, poly-functional and cross-reactive $\mathrm{T}$ cell responses to influenza HA antigens. Clin Immunol 2014; 154:164-177.

21. Bonduelle O, Carrat F, Luyt C-E, Leport C, Mosnier A, Benhabiles $\mathrm{N}$, et al. Characterization of pandemic influenza immune memory signature after vaccination or infection. J Clin Invest 2014; 124:3129-3136.

22. Zens KD, Farber DL. Memory CD4 T cells in influenza. Curr Top Microbiol Immunol 2015; 386:399-421.

23. Liu J, Wu B, Zhang S, Tan S, Sun Y, Chen Z, et al. Conserved epitopes dominate cross-CD8+ T-cell responses against influenza A H1N1 virus among Asian populations. Eur J Immunol 2013; 43:2055-2069.

24. Chiu C, Wrammert J, Li GM, McCausland M, Wilson PC, Ahmed R. Cross-reactive humoral responses to influenza and their implications for a universal vaccine. Ann N Y Acad Sci 2013; 1283:13-21

25. Oertelt-Prigione $\mathrm{S}$. The influence of sex and gender on the immune response. Autoimmun Rev 2012; 11:A479-A485.

26. Lee N, Wong CK, Chan PK, Chan MC, Wong RY, Lun SW, et al. Cytokine response patterns in severe pandemic 2009 H1N1 and seasonal influenza among hospitalized adults. PLoS One 2011; 6:e26050. 
27. Pontoux C, Banz A, Papiernik M. Natural CD4 CD25(+) regulatory $\mathrm{T}$ cells control the burst of superantigen-induced cytokine production: the role of IL-10. Int Immunol 2002; 14:233-239.

28. Wang SM, Tsai MH, Lei HY, Wang JR, Liu CC. The regulatory $\mathrm{T}$ cells in anti-influenza antibody response post influenza vaccination. Hum Vaccin Immunother 2012; 8:1243-1249.

29. Iorio AM, Bistoni O, Galdiero M, Lepri E, Camilloni B, Russano $\mathrm{AM}$, et al. Influenza viruses and cross-reactivity in healthy adults: humoral and cellular immunity induced by seasonal 2007/2008 influenza vaccination against vaccine antigens and 2009 A(H1N1) pandemic influenza virus. Vaccine 2012; 30:1617-1623.

30. Yu X, Zhang X, Zhao B, Wang J, Zhu Z, Teng Z, et al. Intensive cytokine induction in pandemic H1N1 influenza virus infection accompanied by robust production of IL-10 and IL-6. PLoS One 2011; 6:e28680.

31. Itoh Y, Shinya K, Kiso M, Watanabe T, Sakoda Y, Hatta M, et al. In vitro and in vivo characterization of new swine-origin $\mathrm{H} 1 \mathrm{~N} 1$ influenza viruses. Nature 2009; 460:1021-1025.
32. Richards KA, Topham D, Chaves FA, Sant AJ. Cutting edge: CD4 $\mathrm{T}$ cells generated from encounter with seasonal influenza viruses and vaccines have broad protein specificity and can directly recognize naturally generated epitopes derived from the live pandemic H1N1 virus. J Immunol 2010; 185:4998-5002.

33. Sridhar S, Begom S, Bermingham A, Ziegler T, Roberts KL, Barclay WS, et al. Predominance of heterosubtypic IFN-gammaonly-secreting effector memory T cells in pandemic H1N1 naive adults. Eur J Immunol 2012; 42:2913-2924.

34. Ichihashi T, Yoshida R, Sugimoto C, Takada A, Kajino K. Crossprotective peptide vaccine against influenza $A$ viruses developed in HLA-A*2402 human immunity model. PLoS One 2011; 6:e24626.

35. Sridhar S, Begom S, Bermingham A, Hoschler K, Adamson W, Carman W, et al. Cellular immune correlates of protection against symptomatic pandemic influenza. Nat Med 2013; 19:1305-1312. 